\title{
Clinical Characteristics of Human Adenovirus Plastic Bronchitis in 10 Pediatric Cases: A Retrospective Study of Seven Years
}

\author{
Lingjian Zeng ${ }^{1} \cdot$ Jianhua $\mathrm{Wei}^{1} \cdot$ Yuyi Tang ${ }^{1} \cdot$ Enmei Liu $^{1} \cdot$ Qubei Li $^{1}$ (D) $\cdot$ Na Zang $^{1}$ (D)
}

Received: 2 November 2020 / Accepted: 25 April 2021 / Published online: 22 June 2021

(C) Wuhan Institute of Virology, CAS 2021

\section{Dear Editors,}

In recent years, human adenovirus (HAdV) infection has been prevalent in various regions in China (Duan et al. 2021). Research in Guangzhou reported that $9.14 \%$ of fatal pneumonia cases showed HAdV-PCR positive (Ou et al. 2007). The severity and high mortality caused by HAdV have aroused the attention of pediatricians. Plastic bronchitis (PB) is a critical disease characterized by the formation of the casts of the bronchial tree that obstructs the airway, while the pathogenesis is not yet clear. PB is divided into two types according to its pathological manifestations (Seear et al. 1997), in which type I (inflammatory) is composed of fibrin and a large number of inflammatory cells (eosinophils, neutrophils, etc.), and it occurs in infectious disease mostly. Influenza virus and Mycoplasma pneumoniae were the most common pathogens of PB in China (Guo et al. 2017; Ding et al. 2014). So far, adenovirus PB was rarely reported. Herein we summarized a total of 10 cases with adenovirus PB from July 2012 to December 2019 diagnosed in our hospital, hoping to provide a reference for clinical diagnosis and treatment.

There are four males and six females in our study, the age of onset ranged from 10 months to 15 years and 7 months, $80 \%$ of the children were younger than 5 years old. All children had no underlying diseases such as congenital heart disease, asthma or pulmonary cystic fibrosis,

Na Zang

zangna1214@126.com

$\triangle$ Qubei Li

liqubei@126.com

1 Department of Respiratory Medicine, Ministry of Education Key Laboratory of Child Development and Disorders, National Clinical Research Center for Child Health and Disorders, China International Science and Technology Cooperation Base of Child Development and Critical Disorder, Children's Hospital of Chongqing Medical University, Chongqing Key Laboratory of Pediatrics, Chongqing 400014, China but one patient (number 3 in Table 1) had bronchiectasis, bronchial stenosis, and a history of left lower pulmonary lobectomy. All patients had fever and cough, and the peak temperature ranged from $38.6{ }^{\circ} \mathrm{C}$ to $41.0{ }^{\circ} \mathrm{C}$, with an average course of fever being $9.80 \pm 6.563$ days. Eight patients had dyspnea, five had wheezing, and two were dispirited. Reduced breath sounds of the lungs were found in all patients, among which eight showed moist rales or wheezing sounds. Among the 10 patients, five children had a white blood cell count less than $4 \times 10^{9} / \mathrm{L}$ (normal reference range $\left.4 \times 10^{9} / \mathrm{L}-10 \times 10^{9} / \mathrm{L}\right)$, and six children had elevated C-reactive protein (CRP) (normal reference range $<8 \mathrm{mg} / \mathrm{L}$ ), while five patients had significantly increased procalcitonin (PCT) (normal reference range $<0.05 \mathrm{ng} / \mathrm{L}$ ). Five children had elevated transaminase, seven patients had increased lactate dehydrogenase, two patients developed abnormal coagulation function, three patients developed hypoproteinemia and seven patients developed anemia.

To investigate the pathogens in those PB patients, all children underwent sputum and bronchoalveolar lavage fluid (BALF) ELISA test (D3 Ultra DFA Respiratory Virus Screening \& ID Kit, ShangHai, China) for pathogen antigens (respiratory syncytial virus, adenovirus, influenza A virus, influenza $B$ virus, type 1-3 parainfluenza virus) and nucleic acid test for adenovirus [Respiratory Adenovirus DNA Diagnostic Kit (PCR-Fluorescence Probing)], and Mycoplasma pneumoniae [Mycoplasma Pneumoniae DNA Diagnostic Kit (PCR-Fluorescence Probing), Shengxiang Biotechnology, Hunan, China). Serum ELISA tests for enterovirus EV-A71 IgM (EV71 -IgM kit, EI20201207A, Wantai Biopharm, Beijing, China), Coxsackie group B virus IgM (Coxsackie group B virus IgM kit, Beier, Beijing, China), Mycoplasma pneumoniae IgM [Diagnostic Kit for Measurement of Antibodies to Mycoplasma pneumoniae (Fassive Particle Agglutination), SERODIA-MYCO II], Epstein-Barr virus IgM and IgG (EBV IgM kit, E201201BV; EBV IgG Kit, E201201BX, EUROIMMUN PerkinElmer company) antibodies were performed as well. 


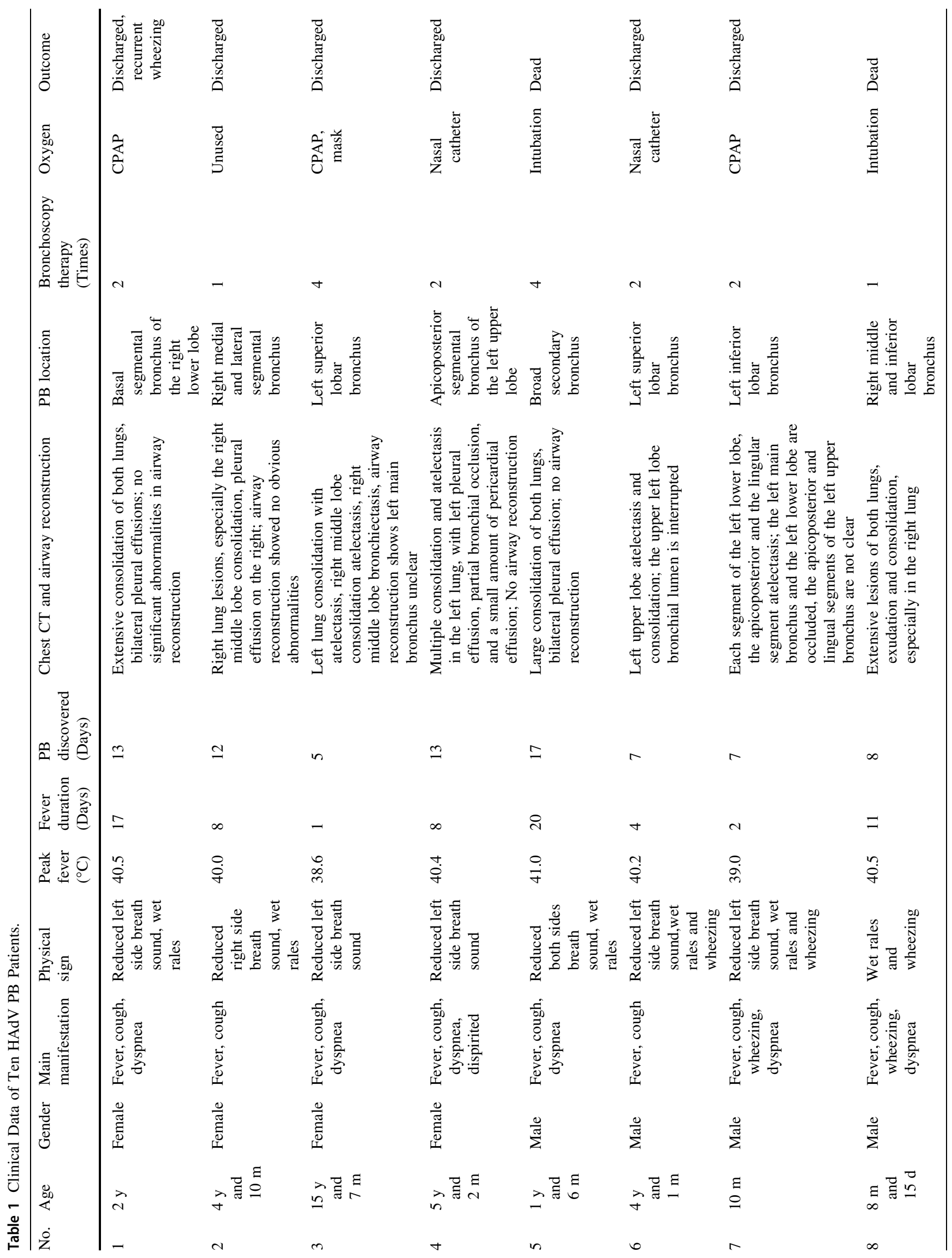




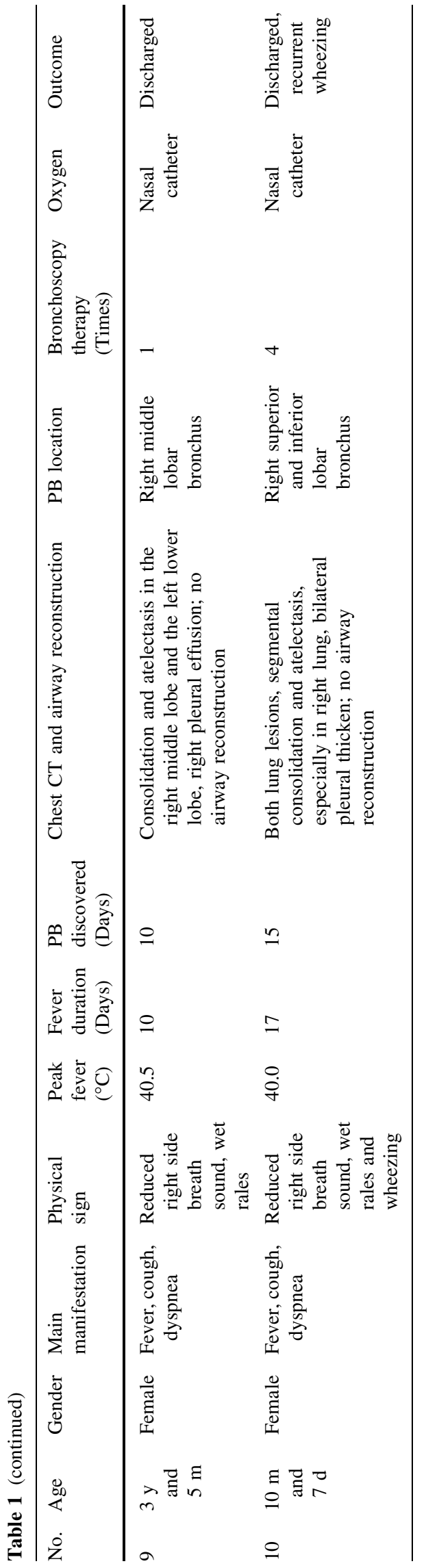

Pathogen tests showed HAdV antigen positive in seven children, while all patients showed HAdV-PCR positive, with the viral load ranged from $9.34 \times 10^{3}$ copies $/ \mathrm{mL}$ to $1.14 \times 10^{8}$ copies $/ \mathrm{mL}$ (Table 2). Seven patients showed bacteria co-infection and the most common pathogen was Streptococcus pneumoniae by sputum culture. Two patients showed co-infection with another virus. There were no Mycoplasma pneumoniae and influenza virus detected.

All chest CT examinations before bronchoscopy showed consolidation or atelectasis (Fig. 1), including four patients with lung consolidation and atelectasis, five with only lung consolidation, and one with only atelectasis, besides, five patients showed pleural effusion. Six patients underwent airway reconstruction, and four of them (66.7\%) showed bronchial occlusion or unclear. Adenovirus and inflammatory mediators can cause edema, hyperemia, and necrosis of bronchial and bronchiolar mucosa, as well as increased mucus secretion thus blocking the lumen (National Health Commission of the People's Republic of China and National Administration of Traditional Chinese Medicine, 2019). Tracheoscopy found that all patients' airway was blocked by branching casts (Fig. 2), and one patient was associated with purulent changes. About 50\% (5/10) of the patients' casts distributed in the right lung, $40 \%(4 / 10)$ distributed in the left lung and $10 \%(1 / 10)$ covered both lungs. And $70 \%$ of the casts involved a single lung lobe, without obvious distribution differences. Cast biopsy from one patient in our group showed a large amount of fibrin and inflammatory exudates, consistent with the performance of type I PB. Five patients underwent cytological examination of alveolar lavage fluid and showed significantly increased nucleated cells (Table 2).

The average time to remove the casts was $10.7 \pm 3.917$ days after the onset of fever. Seven patients performed at least twice tracheoscopy therapy during hospitalization, and one patient experienced four times bronchoscopy because of extensive secondary bronchial obstruction and failed to get a complete removal. Other six patients found no PB recurrence after three days interval of the first time. Two patients were readmitted to the hospital because of atelectasis twenty days after discharge, and no casts were found by bronchoscopy, These results suggest that there is no recurrence of adenovirus PB in short term, but multiple times of bronchoscopy lavage treatments still have a certain effect on the early expansion of atelectasis, the absorption of lung lesions, and the shortening the hospital stay (Zhao et al. 2020).

There is no recommended effective drug for adenovirus pneumonia (National Health Commission of the People's Republic of China and National Administration of Traditional Chinese Medicine, 2019) and current treatment strategies mainly focus on symptomatic and supportive 
care such as oxygen therapy and respiratory support, immunomodulators (such as globulin and glucocorticoids), and bronchoscopy, etc. In our study, 90\% (9/10) of the patients required oxygen therapy, two of them received tracheal intubation and mechanical ventilation, and three required non-invasive ventilation. Some patients' dyspnea was relieved before the removal of casts, which is much less severe compared with previous reports that children with influenza virus PB all require mechanical ventilation about 2.5 days after the onset of disease (Bai et al. 2015). We think it is because the influenza virus had extensive lesion areas including bronchi, bronchiole and alveolar epithelium, and can progress to multiple lung lobes and cause severe damage rapidly, while most of the adenovirus casts in our group have limited lung lobes involvement, so most dyspnea in our group is less severe. The time of PB discovered and duration of mechanical ventilation in our group are similar compared with Mycoplasma pneumoniae PB (Jiang et al. 2019). Antibiotics therapy should be considered when bacterial coinfection is detected. Ten children in our study who had obvious infection-toxic manifestations were considered to have bacterial co-infections or bacterial infections that cannot be completely excluded and were treated with antibiotics. $30 \%$ of the children in our group were treated with intravenous methylprednisolone to reduce inflammation. It is reported that early use of glucocorticoid when it causes obvious systemic inflammatory response has a certain effect on inflammation control and disease relief (Zhang et al. 2016). Intravenous immunoglobulin (IVIG) was used in four patients in our study. All patients underwent bronchoscopy lavage treatment and were treated with $\mathrm{N}$-acetylcysteine in

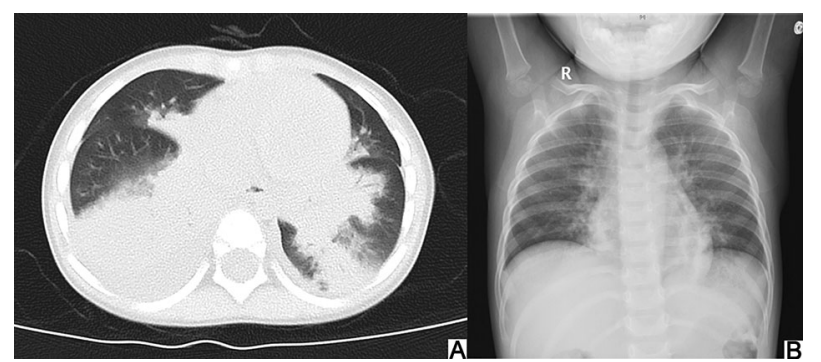

Fig. 1 Chest CT showing consolidation and atelectasis in the inferior lobe of the right lung before bronchoscopy (A) and distinct improvement of chest X-ray after 3 days of bronchoscopy (B).

the trachea through bronchoscopy. Bronchoscopy is currently considered to be the preferred treatment for $\mathrm{PB}$ (Rubin, 2016).

There were eight patients complicated with respiratory failure, one with ARDS, five with liver function impairment, two with myocardial function impairment, two with toxic encephalopathy, and one with secondary hemophilia syndrome. Eight patients were discharged and their average length of hospital stay was $11.38 \pm 3.335$ days. Two children died, one due to the casts couldn't be taken out and resulted in persisting hypoxemia, another died of secondary hemophagocytic syndrome. This mortality of $\mathrm{PB}$ is much higher than previously reported ( $8.0 \%$ to $11.9 \%)$ in China (Liang and Huang, 2016; Zhu et al. 2013). Among the eight survived children, telephone follow-up showed two of them had repeated wheezing after activities, whether adenovirus PB patients have a higher incidence of bronchiolitis obliterans needs to be further studied.

To date, only two cases of adenovirus PB have been reported in the literature (Lu and Zheng 2018; Zhang et al.

Table 2 Pathogen detection in ten adenovirus plastic bronchitis patients.

\begin{tabular}{|c|c|c|c|c|}
\hline No. & $\begin{array}{l}\text { Sputum HAdV load } \\
\text { (copies } / \mathrm{mL} \text { ) }\end{array}$ & $\begin{array}{l}\text { BALF HAdV load } \\
\text { (copies/mL) }\end{array}$ & $\begin{array}{l}\text { BALF nucleated cell count }\left(\times 10^{6} / \mathrm{L} \text {, percentage in }\right. \\
\text { total nucleated cell) }\end{array}$ & Co-infected pathogens \\
\hline 1 & $1.56 \times 10^{7}$ & $6.77 \times 10^{6}$ & 2459 (МØ 69\%, Lymph18\%, Nuet 12\%) & EBV \\
\hline 2 & $2.44 \times 10^{7}$ & $1.27 \times 10^{5}$ & Undone & Streptococcus pneumoniae \\
\hline 3 & $9.34 \times 10^{3}$ & Undone & 42,800 (Nuet 87\%, MØ 5\%, Lymph 3\%) & Pseudomonas aeruginosa \\
\hline 4 & $1.62 \times 10^{6}$ & $3.16 \times 10^{5}$ & 19,680 (Lymph 52\%, Mø 15\%, Nuet 14\%) & $\begin{array}{l}\text { Streptococcus pneumoniae, } \\
\text { EBV, CVB }\end{array}$ \\
\hline 5 & $1.14 \times 10^{8}$ & $5.19 \times 10^{7}$ & Undone & Haemophilus influenzae \\
\hline 6 & $2.93 \times 10^{4}$ & Undone & 2000 (Lymph 18\%, Nuet 52\%, MØ 23\%) & Moraxella catarrhalis \\
\hline 7 & $6.98 \times 10^{6}$ & Undone & 24,800 (Nuet 37\%, Lymph 32\%, MØ 29\%) & Not found \\
\hline 8 & $1.02 \times 10^{7}$ & Undone & Undone & Escherichia coli \\
\hline 9 & $\begin{array}{l}\text { Negative (antigen- } \\
\text { positive) }\end{array}$ & $1.18 \times 10^{6}$ & Undone & Streptococcus pneumoniae \\
\hline 10 & $\begin{array}{l}\text { Undone (antigen- } \\
\text { positive) }\end{array}$ & $\begin{array}{l}\text { Positive(viral load } \\
\text { unknown) }\end{array}$ & Undone & Not found \\
\hline
\end{tabular}

$M \emptyset$, Macrophage; Lymph, Lymphocyte; Nuet, Neutrophile; $C V B$, Coxsackie group B virus. EBV, Epstein-Barr virus. 
Fig. 2 The appearance of HAdV PB under bronchoscopy (A), branching cast taken out in HAdV PB (B), and large amounts of fibrin and inflammatory cells infiltrated in histopathology (C).

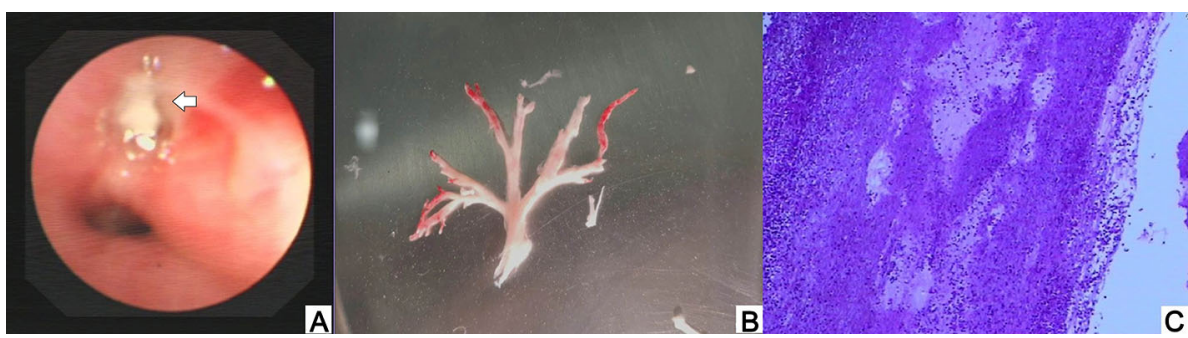

2020) and no deaths were reported. One of the two cases was induced by HAdV-7 and received extracorporeal membrane oxygenation (ECMO) treatment for one week, the type II respiratory failure was corrected after removing the casts through bronchoscopy, which suggested that adenovirus PB can be improved with early active treatment and timely removal of casts when no serious secondary complications occurred.

In conclusion, HAdV pneumonia can cause PB. When dyspnea occurs, especially refractory hypoxemia accompanied with reduced respiratory sounds, consolidation or atelectasis in imagines and abnormalities in airway reconstruction, HAdV PB should be highly considered. The timely application of bronchoscopy plays an important role in disease recovery and prognosis.

Acknowledgements We would like to acknowledge the assistance of the patients and their caregivers involved in the study. This study was supported by the Education Committee of Chongqing, China for Young Scholars (Chongqing Education Department [2019] No.13), Millions Talent Projects of Chongqing (Chongqing Commission [2018] NO.187), and the Foundation of Children's Hospital of Chongqing Medical University.

\section{Compliance with Ethical Standards}

Conflict of interest The authors declare that they have no conflict of interest.

Human Rights Statement Ethical approval was provided by the Institutional Review Board of the Children's Hospital of Chongqing Medical University ([2019], No. 167). Informed consent was obtained from the parent or guardian of all participants.

\section{References}

Bai K, Lin XJ, Liu CJ (2015) Critical influenza A complicated with plastic bronchitis in children: 15 cases report. Chongqing Med Univ 40:140-143
Ding XF, Zhong LL, Zhang B, Lin L, Huang H, Liang M (2014) Clinical features and pathogens of plastic bronchitis in children: an analysis of 9 cases. Chin J Contemp Pediatr 16:729-733

Duan Y, Li C, Deng L, An S, Zhu Y, Wang W, Zhang M, Xu L, Xu B, Chen X, Xie Z (2021) Genetic analysis of human adenovirus type 7 strains circulating in different parts of China. Virol Sin. https://doi.org/10.1007/s12250-020-00334-y

Guo YS, Zou YX, Zhai J, Zhao L, Shen Y, Guo R, Huang B (2017) Clinical analysis of type I plastic bronchitis in 73 children. J Tianjin Med Univ 23:422-425

Jiang LL, Wan J, Suo FT, Tang L, Li YY, Tian XY, Zhang GL, Luo ZX (2019) Clinical analysis of Mycoplasma pneumoniae plastic bronchitis in 5 children. Clin Pediatr 37:273-276

Liang JM, Huang XW (2016) Clinical analysis of 50 children with plastic bronchitis. J Clin Pulm Med 21:1571-1574

Lu ZW, Zheng YJ (2018) Plastic bronchitis associated with adenovirus infection. Lancet Infect Dis 18:474

National Health Commission of the People's Republic of China and National Administration of Traditional Chinese Medicine (2019) Guideline for diagnosis and treatment of adenovirus pneumonia in children, 2019 version. Chin J Clin Infect Dis 3:161-166

Ou ZY, Zeng QY, Wang FH, Xia HM, Lu JP, Xia JQ, Gong ST, Deng L, Zhang JT, Zhou R (2007) Retrospective study of adenovirus in autopsied pulmonary tissue of pediatric fatal pneumonia in South China. BMC Infect Dis 8:122

Rubin BK (2016) Plastic Bronchitis. Clin Chest Med 37:405-408

Seear M, Hui H, Magee F, Bohn D, Cutz E (1997) Bronchial casts in children: a proposed classification based on nine cases and a review of the literature. Am J Respir Crit Care Med 155:364-370

Zhang MZ, He HY, Lv B, Li LZ, Xuan RH, He JJ (2016) Clinical study of adenovirus infection sepsis hormone therapy. Guide China Med 14:25-26

Zhang FZ, Qin L, Yuan JX, Tang LF (2020) Plastic bronchitis due to adenoviral infection: a case report. BMC Pediatr 20:61

Zhao YQ, Dai XM, Ji JZ, Cheng P (2020) Bronchial lavage under fiberoptic bronchoscopy in the treatment of severe pulmonary infection. Pak J Med Sci 36:396-401

Zhu CM, Cao L, Chang L, Zhang Q, Wang F, Ren XX (2013) Report of 5 cases of pediatric plastic bronchitis and review of related literature. Chin J Gen Pract 12:911-913 